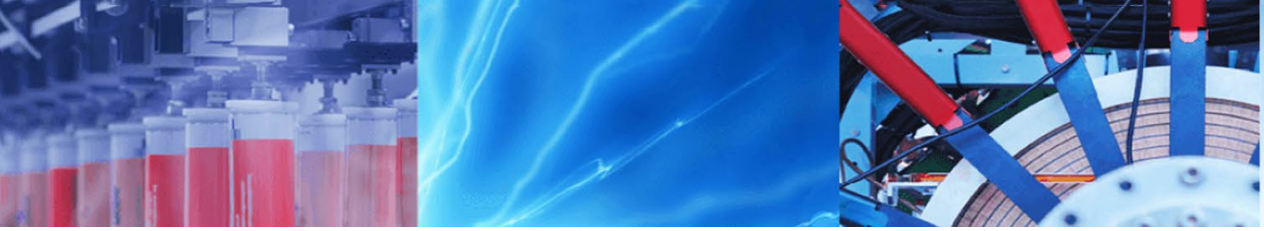

Research Article

\title{
Distance discount in the green vehicle routing problem offered by external carriers
}

\author{
Mahmood Nosrati ${ }^{1}$. Alireza Arshadi Khamseh ${ }^{2}$
}

Received: 27 April 2020 / Accepted: 22 July 2020 / Published online: 28 July 2020

(c) Springer Nature Switzerland AG 2020

\begin{abstract}
This study suggested a model for the Green Vehicle Routing Problem (GVRP) regarding distance discount strategy. For the first time,we defined distance discount strategy based on intervals for a vehicle routing problem. The proposed model attempts to minimize the total cost of the system by selecting the different vehicles and paths that have time and fuel constraints while external carriers offer the distance discount. This model is formulated by mixed-integer linear programming and classified as NP-hard. The feasibility of this issue depends on the location of customers and fuel stations. Two metaheuristic algorithms such as tabu search (TS) and simulated annealing (SA) have been developed beside the exact method, and the efficiency of our metaheuristic methods have been examined through 40 benchmark instances of different sizes and 16 randomly generated numerical instances. Computational results show superior performance of the simulated annealing in comparison with the tabu search for test problems and the cost efficiency owing to the distance discount from the organization's outlook.
\end{abstract}

Keywords Transportation cost discount · Distance discount strategy · Green vehicle routing problem · Tabu search . Simulated annealing

\section{Introduction}

Vehicle Routing Problem (VRP) is one of the crucial problems in transportation, logistics, and supply chain management. In business activities, Logistics and distribution are known as essential sections because often associated with inventory and production decisions, and deliveries' costs are a critical part of the total logistics costs [1]. The VRP is a familiar optimization problem in which the cost of transportation is minimized by determining the desired routes [2]. In VRP, a fleet of vehicles start its routes from a depot and return to the depot again after providing services to the specific customers' collections and completing their work [3].
Today, one of the most critical threats on earth is the problem of climate change. Toxic gases such as carbon dioxide, carbon monoxide and several other greenhouse gases are among the most significant causes of these threats [4]. A significant amount of total greenhouse gas emissions is related to carbon emissions in the transportation sector. Measuring the environmental influences of various distribution policies, decreasing energy usage, decreasing waste, and managing its treatment are such as green logistics activities [5]. Lin et al. [6] reviewed VRPs and separated them into two broad categories: Traditional VRP and GVRP. GVRP is a kind of routing problem that coordinates environmental and economic impressions and includes issues such as vehicles with multiple fuels and alternative fuel stations $[2,7]$.

\footnotetext{
$\triangle$ Alireza Arshadi Khamseh, akhamseh@gelisim.edu.tr; Mahmood Nosrati, mahmoodn221@gmail.com | ${ }^{1}$ Department of Industrial Engineering, Faculty of Engineering, Kharazmi University, Tehran, Iran. ${ }^{2}$ Department of International Logistics and Transportation, Faculty of Economics Administration and Social Sciences, Istanbul Gelisim University, Istanbul, Turkey.
} 
This article is an extension of the green vehicle routing problem. In this case, external carriers are working to gain more customers by offering discounts on traveled distance. The sharing economy, as an exciting subject, leads to outsourcing logistics processes to third-party companies and can be useful to cost reduction and make more effective use of resources in transport and logistics. The findings [8] indicate the magnificent potential to share sources in transportation. In this case, companies use external carriers to deliver their products. On the other hand, carrier companies are also trying to attract more customers in different ways. One of these ways is to offer distance discounts.

Distance is one of the most critical factors in transportation costs. The costs of transportation are different by traveled distance [9]. The cost of transportation depends on factors such as fuel consumption, workforce costs, depreciation costs, and the maintenance of vehicles. Some of them are not directly related to the traveled distance. Therefore, carriers consider lower shipping costs for longer distances. This study assumed that the cost of transport changes over different distance intervals and decreases with increasing distance. So that longer-distance tours get more discounts and lower shipping costs.

Figure 1 depicts an instance for two definitions of GVRP; with or without distance discount. In this example,we encounter a 10 percent discount for distances over $500 \mathrm{~km}$. For simplification of the problem, cost for the distance unit has been considered one.Here,the total traveling distance in the non-discount and distance discount mode are 1060.27 and 1083.32 respectively when the related costs are $1060.27,1021.30$ in the same order.As it is obvious here we have 23.05 unit reduction in total cost by means of distance discount. Table 1 shows the details of the results.

Distance discounts could apply to different vehicle routing problems, but the existence of fuel and time constraints in GVRP will help to make the answers more realistic.

This paper is prepared as follows. Section 2 presents the literature review on GVRP and transportation cost discount. Section 3 introduces the problem definition and assumptions. Mathematical formulations are detailed in Sect. 4. Section 5 describes solving methods and computational results will be illustrated in Sect. 6. Finally, in Sect. 7 conclusions and further research plans will be regarded.
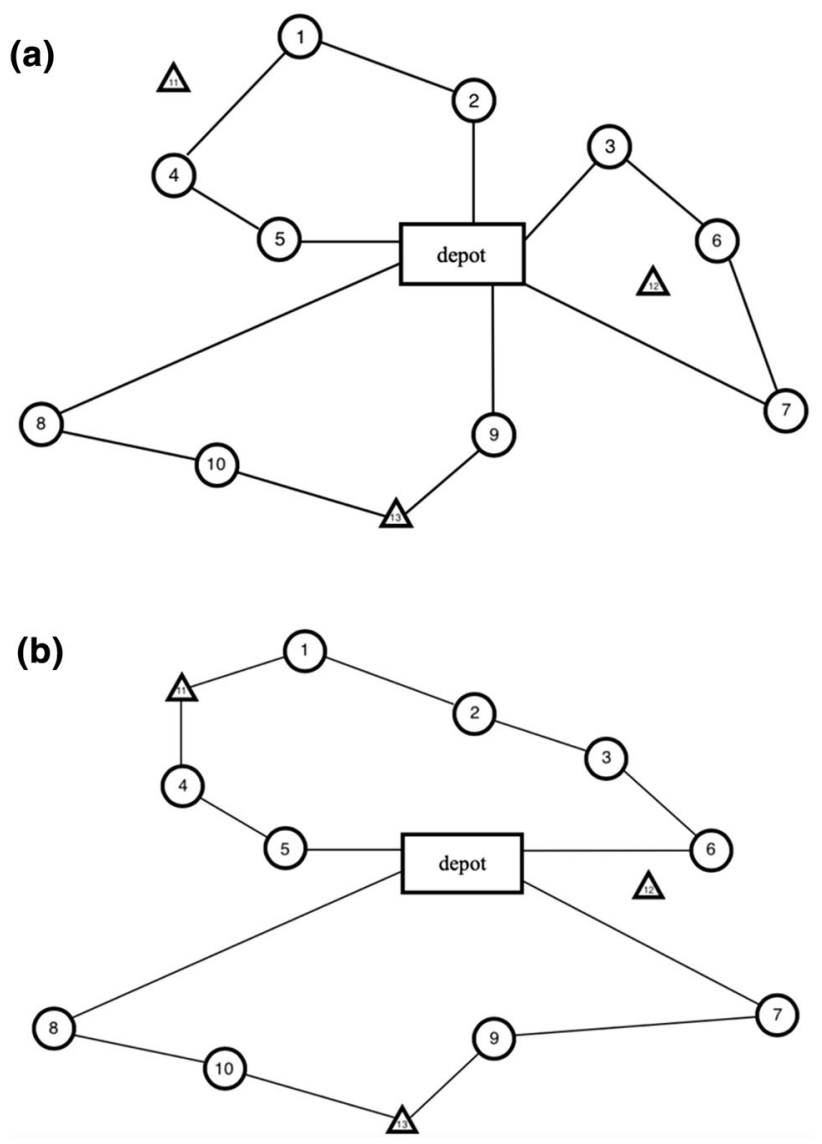

Fig. 1 GVRP without discount (a) and GVRP with distance discount (b)

Table 1 Detail of GVRP with distance discount example

\begin{tabular}{lll}
\hline & $(\mathrm{a})$ & $(\mathrm{b})$ \\
\hline Customer sequence of each & $0,3,6,7,0$ & $0,5,4,11,1,2,3,6,0$ \\
route & $0,2,1,4,5,0$ & $0,8,10,13,9,7,0$ \\
& $0,8,10,13,9,0$ & \\
Distance of each rout & 312.19 & \\
& 328.99 & 463.16 \\
Total distance & 419.09 & 620.16 \\
Cost of each rout & 1060.27 & 1083.32 \\
& 312.19 & 463.16 \\
& 328.99 & 558.14 \\
Total cost & 419.09 & \\
\hline
\end{tabular}




\section{Literature review}

\subsection{Green vehicle routing problem}

In existing research in VRP, minimizing fuel consumption looks infrequent. Kara et al. (2007) assumed a more pragmatic transportation cost, which is influenced by vehicle burden as well as distance traveled. They defined a new cost function of CVRP with energy minimizing that contains the total load (including empty vehicle weight) and the arc length. However, they do not provide formulation details of fuel use [10]. Xiao et al. (2012) stated that among the total cost of oil-based shipping, fuel cost values play a meaningful role. Furthermore, the amount of fuel used during the operation of a fleet is a more significant concern than the total distance traveled in transportation companies. They provided a formulation of fuel consumption and suggested a Fuel Consumption Rate (FCR), which extends CVRP intending to minimize fuel use. They considered both covered distance and load as factors that define fuel costs [11].

Gon calves et al. investigated a pickup and delivery vehicle routing, and a fleet composed of electric vehicles and traditional vehicles. They stated time constraints and added additional time to charge electric vehicle batteries besides considering capacity limitation. In their model, vehicles can be recharging at any time during a route. They have not explicitly regarded the location of the recharging station in their model [12]. Conrad and Figliozzi proposed the VRP with recharging vehicles that vehicles can be recharging at some customer places while servicing customers. This work adds extra time as the penalty to total route time [13].

Erdoğan and Miller-Hooks were the prime scholars that studied VRP with the possibility of refueling in the routes. They assumed the refueling station, service time for every node, and the maximum duration constraint in the problem; that way, vehicles can refuel on each tour to increase the distance traveled. They denoted this problem as a green vehicle routing. Their model attempts to minimize the total distance and tries to eliminate run out of fuel risk [2]. Related to their scholarship, Schneider et al. extended the GVRP with electric vehicles, time windows, and refueling stations [7]. Felipe et al. developed the model presented by Erdoğan and Miller-Hooks with multiple recharging technologies and allowed partially refueling at the stations [14]. Bruglieri et al. considered the capacity limitation of fueling stations in GVRP. They introduced Two Mixed Integer Linear Programming models based on arc and path variables [15]. Zhang et al. considered the limitation on loading capacity on GVRP that Leads to difficulty in route designing. They used two-phase heuristic and the meta-heuristic based on an ant colony system to solve the model [16]. Andelmin and Bartolini proposed an exact algorithm for solving the GVRP. They modeled the GVRP as a set partitioning problem and showed the capability of solving instances with up to 110 customers [17]. They proposed a multi-start local search (MSLS) algorithm and introduced three phases. The first and second phases make, improve, and save new solutions. In the third phase, a set partitioning problem is solved [18]. Peng et al. proposed an efficient algorithm as the memetic algorithm (MA) to solve GVRP. They used reinforcement learning to control neighborhood moves and supervise the search [19].

\subsection{Discount in transportation costs}

Transport is a substantial part of supply chain activities. Attention to transportation costs in system decisions can decrease the entire cost of the supply chain [20]. Discount strategies are primary marketing mechanisms for persuading customers to more extent procurement. Organizations usually encounter quantity discounts from suppliers and freight discounts from shippers [21]. Also, external common carriers and subcontractors may use discount policies to reach a higher customer rate that drives to decrease in entire transportation costs [22]. Accordingly, the operational costs of an external carrier can decrease and lead to achieve a competitive advantage. Consequently, the carrier will reduce delivery costs and offer discounts [23].

Tsao et al. [24] introduced an integrated facility location and inventory allocation problem in a multi-echelon network design considering transportation cost discounts. They assumed that the shipping cost contains a fixed cost and an added variable cost based on distance and considered two types of discounting policies concurrently: quantity discounts and distance discounts. They considered distance discounts for transportation costs between regional distribution centers and retailers. Because the fuel usage pertains to distance and distance cost is a function of factors such as labor costs, therefore, if the transportation distance were longer, the cost of transporting would be lower. Also, they studied quantity discounts between general delivery centers and local delivery centers and that is the first essay that studied two different shipping discounts together in the supply chain network design problem.

Karimi et al. studied a mixed production-distribution planning in definitive, multi-product, and multi-echelon networks to minimize the total shipping costs and transfer time, subject to fulfilling retailer demands and capacity limitations. Their model included vehicles' fixed cost, routing decisions, and quantity and distance discounts on all levels of the supply chain's transportation costs. In their 
paper, the transportation costs commanded by third-party logistics companies are related to shipment quantities and distances [25].

Gahm et al. [23] extended the VRP with private fleet and common carriers by three perspectives: two exclusive rental options as additional options for subcontracting, a more realistic cost function regarding distances, and volume discounts suggested by the common carriers. Stenger et al. [26] proposed the prize-collecting model in the single and multi-depot variant of the vehicle routing that has the possibility of outsourcing clients instead of serving them with the private fleet to model an extremely relevant planning task in small pack shipping, whereby subcontractor provided the discount that follows a non-linear cost function for more substantial amounts of load.

Here we defined distance discount from the organizational viewpoint which is the first to our knowledge and has not been clarified before in detail in Green Vehicle Routing Problems.

\section{Problem definition}

In today's competitive world, giving services that meet expectations and have lower prices can provide a competitive advantage and make companies more popular among customers. Therefore, in some situations, distributors and carriers offer their services at lower prices through methods such as discount strategies. In our case, distribution in the GVRP would be done by external carriers (subcontractors) in a way that every subcontractor is seeking to attract more customers by offering discounts.

GVRP searches a set of vehicle tours to minimize total travel costs in which vehicles start to move from the depot, tour the set of customers, and end their route at a depot within a designated time according to maximum time constraint. Also, due to fuel constraints, each vehicle can refuel along the route. It is assumed that refueling stations and also depots can be used as a refueling station and have unlimited capacity. Also, during refueling, the tank is filled up to full capacity. In this problem, the fleets use similar vehicles.

The vehicles have no limitation on the number of refueling stops in each tour. Each node has service time, and every pair of nodes has a specific travel time and distance and the speed of travel is constant for every route.

There is a distance discount in the transportation system, in which the transportation cost would be increased regarding the rising of the distance, but not completely consistent with the rise of the distance and for being closer to the realistic conditions, we proposed a stepwise discount model. To model the distance discount

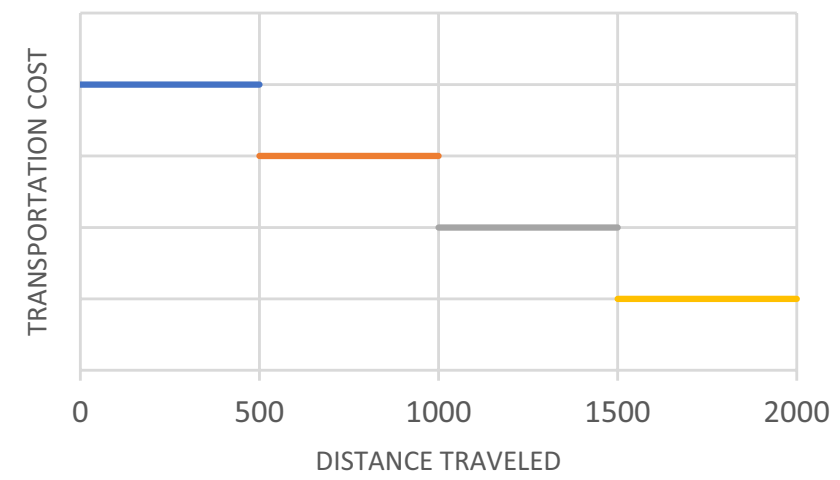

Fig. 2 Transportation cost in different discount intervals

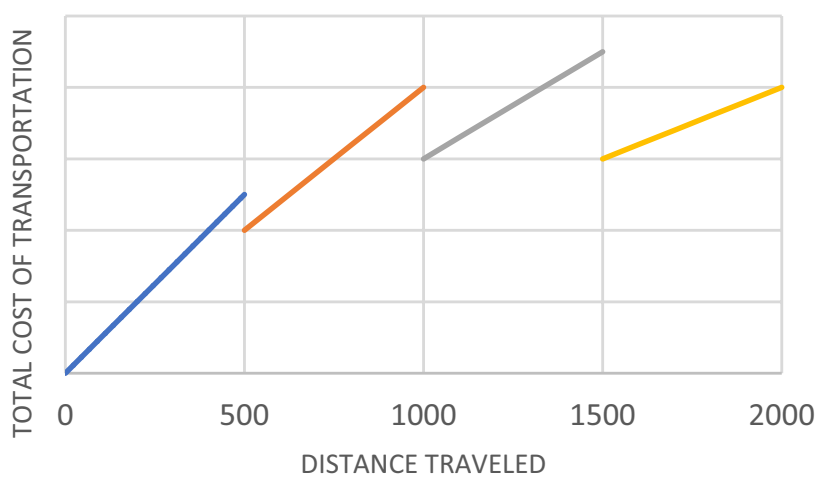

Fig. 3 Total transportation cost in different discount intervals

intervals, the model of all unit discount strategies has been used.

As shown in Fig. 2, transportation cost is different in each distance range, and would be decreased with increasing distance traveled. Notably, a discount interval should be activated only when its previous interval reaches its maximum distance, and at the same time, just one of the lines would be considered. Figure 3 illustrates distance discount intervals and their impact on total transportation costs.

\section{Mathematical formulation}

Following Erdoğan and Miller-Hooks [2], the green vehicle routing problem defined on an undirected complete graph $G=(V, E)$ consists of vertices representing customer locations $\mathrm{I}=\left\{v_{1}, v_{2}, \ldots, v_{n}\right\}$, recharging stations $F=\left\{v_{n+1}, v_{n+2}, \ldots, v_{n+f}\right\}$, and a depot $v_{0}$. The set $\mathrm{E}=\left\{\left(v_{i}, v_{j}\right): v_{i}, v_{j} \in V, i<j\right\}$ corresponds to the edges connecting the vertices of set $V$ are defined as the entire set $V=\left\{v_{0}\right\} \cup I \cup F=\left\{v_{0}, v_{1}, v_{2}, \ldots, v_{n+s}\right\}$. The two main 
parameters in distance discount modeling are $n_{s}$ and $c_{s}$. The parameter $n_{s}$ indicates the distance range at which the discount percentage changes, when $c_{s}$ determines this discount percentage. In this notation, $s$ shows the breakpoints index. The variable $I_{k}$ is related to the traveled distance of the vehicle $k$. When this distance is placed in each of the discount ranges, the whole route of the vehicle includes the discount. In this way, when the distance traveled by the vehicle $\left(I_{k}\right)$ passes $\left(n_{s}\right)$, the relevant discount percentage $\left(c_{s}\right)$ is applied for the whole route $k$. In the following, the notations used in formulating the GVRP have been introduced.

\subsection{Index}

\section{Set of customers}

$I_{0}$ Set of customers and warehouses

$F \quad$ Set of refueling stations

$F_{0} \quad$ Set of refueling and storage stations

$V$ Set of warehouses, customers and refueling stations

$K$ Set of vehicles

$S$ Breakpoints $s=(1,2 \ldots, w)$

\subsection{Parameters}

$m \quad$ Allowed number of vehicles

$r \quad$ Fuel consumption rate

$Q \quad$ Vehicles tank capacity

$P \quad$ Service time

$T_{\max }$ Maximum allowed time for each route

$d_{i j} \quad$ Distance between $\mathrm{i}$ and $\mathrm{j}$ nodes

$t_{i j} \quad$ The time interval between nodes $\mathrm{i}$ and $\mathrm{j}$

$c_{s} \quad$ Discount percentage for any breakpoint

$n_{s} \quad$ The discount breakpoint s

\subsection{Positive variables}

$y_{j} \quad$ The remaining fuel level at $j$ point. This amount is readjusted at the refueling and storage stations to $Q$

$\tau_{j} \quad$ Vehicle's arrival time to the point $\mathrm{j}$

$I_{k}$ The length of the route traveled by the vehicle $k$

\subsection{Binary variables}

$x_{i j k} \quad$ Equals to 1 if vehicle $\mathrm{k}$ has a route between $\mathrm{i}$ and $\mathrm{j}$, elsewhere equeals to 0 . $x_{i j k s}^{\prime} \quad$ Equals to 1 if the vehicle $k$ has a route between $\mathrm{i}$ and $\mathrm{j}$ at the discount breakpoint $\mathrm{s}$, and elsewhere equeals to 0

\subsection{Mathematical model}

$\min Z=\sum_{i \in V} \sum_{j \in V} \sum_{k} \sum_{s}\left(1-c_{k s}\right) d_{i j} x_{i j k s}^{\prime}$

$\sum_{j \in V} \sum_{k} x_{i j k}=1 \forall i \in I$

$j \neq i$

$\sum_{j \in V} \sum_{k} x_{i j k} \leq 1 \forall i \in F$

$j \neq i$

$\sum_{i \in V} x_{j i k}-\sum_{i \in V} x_{i j k}=0 \forall j \in V, k$

$j \neq i \quad j \neq i$

$\sum_{\substack{j \in V \\ j \neq 0}} \sum_{k} x_{0 j k} \leq m$

$j \neq 0$

$\tau_{j} \geq \tau_{i}+\left(t_{i j}+p\right) x_{i j k}-T_{\max }\left(1-x_{i j k}\right)$

$\forall i \in V, k, j \in V \backslash\{0\}$ and $\neq j$

$0 \leq \tau_{0} \leq T_{\max }$

$t_{0 j} \leq \tau_{j} \leq T_{\text {max }}-\left(t_{j 0}+p\right) \forall j \in V \backslash\{0\}$

$y_{j} \leq y_{i}-r . d_{i j} x_{i j k}+Q\left(1-x_{i j k}\right)$

$\forall j \in l, i \in V$, kandi $\neq j$

$y_{j}=Q \forall j \in F_{0}$

$y_{i} \geq \sum_{j \in F_{0}} r . d_{i j} x_{i j k} \forall i \in V, k$ 


$$
\begin{aligned}
& \sum_{\substack{j \in V \\
j \neq i}} x_{i j k} \leq 1 \forall i \in V, k \\
& \sum_{k} x_{i j k} \leq 1 \forall i \in V, j \in \text { Vandi } \neq j \\
& \sum_{s} x_{i j k s}^{\prime}=x_{i j k} \forall i \in V, j \in V, k a n d i \neq j \\
& \sum_{\substack{i \in V \\
i \neq j}} \sum_{j \in V}\left(x_{i j k} * d_{i j}\right)=I_{k} \forall k \\
& I_{k} \geq x_{i j k s}^{\prime} \cdot n_{k s-1} \forall i \in V, j \in V, k, \text { sand } i \neq j \\
& x_{i j k}, x_{i j k s}^{\prime} \in\{0,1\} \forall i, j, k, s
\end{aligned}
$$

The objective function (1) is defined to minimize the total transportation cost. Constraint (2) states that each customer is met precisely once, and constraint (3) states that each refueling center must be met precisely one time. Route continuity is guaranteed by the constraint (4). The constraints (5) and (6) describe the maximum number of routes. Constraint (7) controls the arrival time to any point. Constraints (8) and (9) guarantees that any vehicle will not return to the central warehouse after the indicated time. The fuel level of the vehicle is ensured by the constraint (10) at the entrance of any point. Constraints (7) and (10), in addition to the time and fuel constraints, ensure that no sub-tour creates. Constraint (11) places the level of fuel at the exit of the warehouse and refueling centers equal to Q. The constraint (12) ensures that the remaining fuel is sufficient to reach the central warehouse or refueling centers. Constraints (13) and (14) prevent the assignment of a route to more than one vehicle. Constraint (15) determines the discount rate for a specific path. Within the constraint (16), the length of each route will be determined. Constraint (17) specifies that only one discount interval will be considered, in this equation when the traveled distance of one route $\left(I_{k}\right)$ would be more than a distance discount breakpoint $\left(n_{s}\right)$, the equation forces all $x_{i j k s}^{\prime}$ related to route $k$ to take the same $s$ index and makes the discount percentage for the whole route equeal.Finally, Constraint (18) shows the range of variables.

\section{Solution approach}

Vehicle routing problems included optimization problems correlated by mathematics, economics, computer science, and operations research. Since the VRPs are identified as NP-hard problems [27], they are hard and time-consuming to solve. Also, the GVRP and presented model, as particularized cases of the VRP, are in this category as well [2].

Elshaer and Awad [28], in an article, reviewed metaheuristic algorithms for solving the vehicle routing problem and its variants. In their review, $63.7 \%$ were single-based algorithms such as TS, VNS, LNS, SA, ILS, GRASP, and GLS algorithms, and the rest were population-based such as evolutionary computation and swarm intelligence. Their results showed that TS is used for more than 30\% and is the most popular. Also, simulated annealing, with $12.2 \%$, is one popular algorithm to solve the vehicle routing problem.

In this paper, we used both mentioned meta-heuristic methods to optimize the model besides the exact method. These algorithms seek to find the optimal solutions close to the exact method and can be useful for large size models. In metaheuristic algorithms, solutions are not necessarily optimum, and generally, these methods assist managers in decision-making in complicated problems by giving approximate solutions [29].

\subsection{Tabu search}

Tabu Search (TS) was initially developed by Glover and Laguna [30] and is a memory-based and neighborhood search method that avoids local explorations around a local optimum. One of solutions is to retain recent moves attributes or past made solutions in a tabu list. That way, if the algorithm tries to move to a solution that is in the tabu list, the movement is prevented, and other solutions examined. Nevertheless, this is not a permanent feature, and it can be disregarded when some purpose criteria are fulfilled. One widespread example is the objective function that is the best value ever detected. One solution can be accepted if this condition is met [31].

This algorithm works as follows. Create the current solution to start. Generate neighboring solutions based on specified neighborhood structures and evaluate them. Found the best among neighborhood solutions, which is better than the current solution and set it as the new current solution. The procedure repeats, and when the current solution is better than all its neighborhood solutions, 
Table 2 Tabu search algorithm trend

Initialization;

Input parameters;

Create an initial solution randomly;

Repeat

Generate neighborhood solutions;

Evaluate neighbors and specify the best one;

If the best neighboring solution be better than the current solution, accept it as the current solution, and if not, accept the best of forbidden solutions;

Update the tabu list;

Until ending condition is met;

Table 3 Simulated annealing algorithm trend

\begin{tabular}{l} 
Initialization; \\
\hline Input parameters; \\
Create an initial solution randomly; \\
Repeat \\
Generate a neighborhood solution; \\
Evaluate the neighbor solution; \\
If the neighboring solution be better than the current solution, \\
accept it as the current solution, and if not, accept it by probabil- \\
ity function; \\
Reduce the temperature; \\
Until ending condition is met;
\end{tabular}

the local search stops, and the current solution is a local optimum [32]. In order to prevent cycling, recently visited solutions are placed in a tabu list and forbidden for a number of iterations [33]. The tabu search trend shown in Table 2.

\subsection{Simulated annealing}

Kirkpatrick et al. [34] proposed simulated annealing for optimization problems for the first time. Since then, this algorithm has been widely used in complicated optimization [35]. SA is a local search-based algorithm that tries to find a global optimum by a mechanism to escape from the local optimum [24]. Simulated annealing is an algorithm
Table 5 Percentage of discount for different intervals

\begin{tabular}{lllll}
\hline Distance & $0-500$ & $500-1000$ & $1000-1500$ & $1500-2000$ \\
\hline Discount percentage & 0.0 & 0.10 & 0.15 & 0.20 \\
\hline
\end{tabular}

based on iron gradually cooling down from melting point to the freezing temperatures.

Whatever iron has the less temperature leads to more limited molecular movement in iron particles. In this algorithm, temperature associates with acceptance probability, and this probability reduces by lowering the temperature over time. This feature helps this algorithm to have less vulnerability to trapped in local optimum solutions [36].

SA can solve VRP and related problems effectively [37]. SA starts the search from an initial solution to find better solutions. If the new solution is better than the current solution, it will replace the current solution, and if it is worse than the current solution, it will be accepted based on a function associated with probability and temperature. In some conditions accepting such solutions helps SA not to be trapped in local optimum [38]. SA uses parameters such as initial temperature, allowed iteration numbers at each temperature, the cooling rate, and the final temperature or ending condition [39]. Table 3 shows the simulated annealing trend.

\section{Computational results}

The proposed mathematical model is linear mixed-integer programming. Computational experiments performed on a system with a $1.3 \mathrm{GHz}$ Intel Core i5 processor with $4 \mathrm{~GB}$ of RAM and Windows 64-bit. In the exact method, the model was coded in the GAMS version 24 and has been solved using the CPIEX solver. Due to the NP-hard of the problem, the time of the exact solution increases dramatically in large dimensions. Therefore, we used the tabu search and simulated annealing to solve the model, especially in large sizes. The introduced meta-heuristic algorithms were coded in version 2016 of MATLAB.

To demonstrate the efficiency of proposed algorithms, we examined them on a particular instance in the GVRP (denoted by AB) that benchmark results of that can be found in the literature. As well as, to indicate the
Table 4 Optimal parameters of TS and SA algorithms

\begin{tabular}{|c|c|c|c|c|c|c|}
\hline \multirow{2}{*}{$\begin{array}{l}\text { Algorithm } \\
\text { Parameter }\end{array}$} & \multicolumn{3}{|l|}{ TS } & \multicolumn{3}{|l|}{ SA } \\
\hline & in & $T L$ & Lim & maxit $_{\text {in }}$ & $\alpha$ & $t_{0}$ \\
\hline Optimal & $2 n$ & $n$ & 50 & 80 & .98 & 100 \\
\hline
\end{tabular}




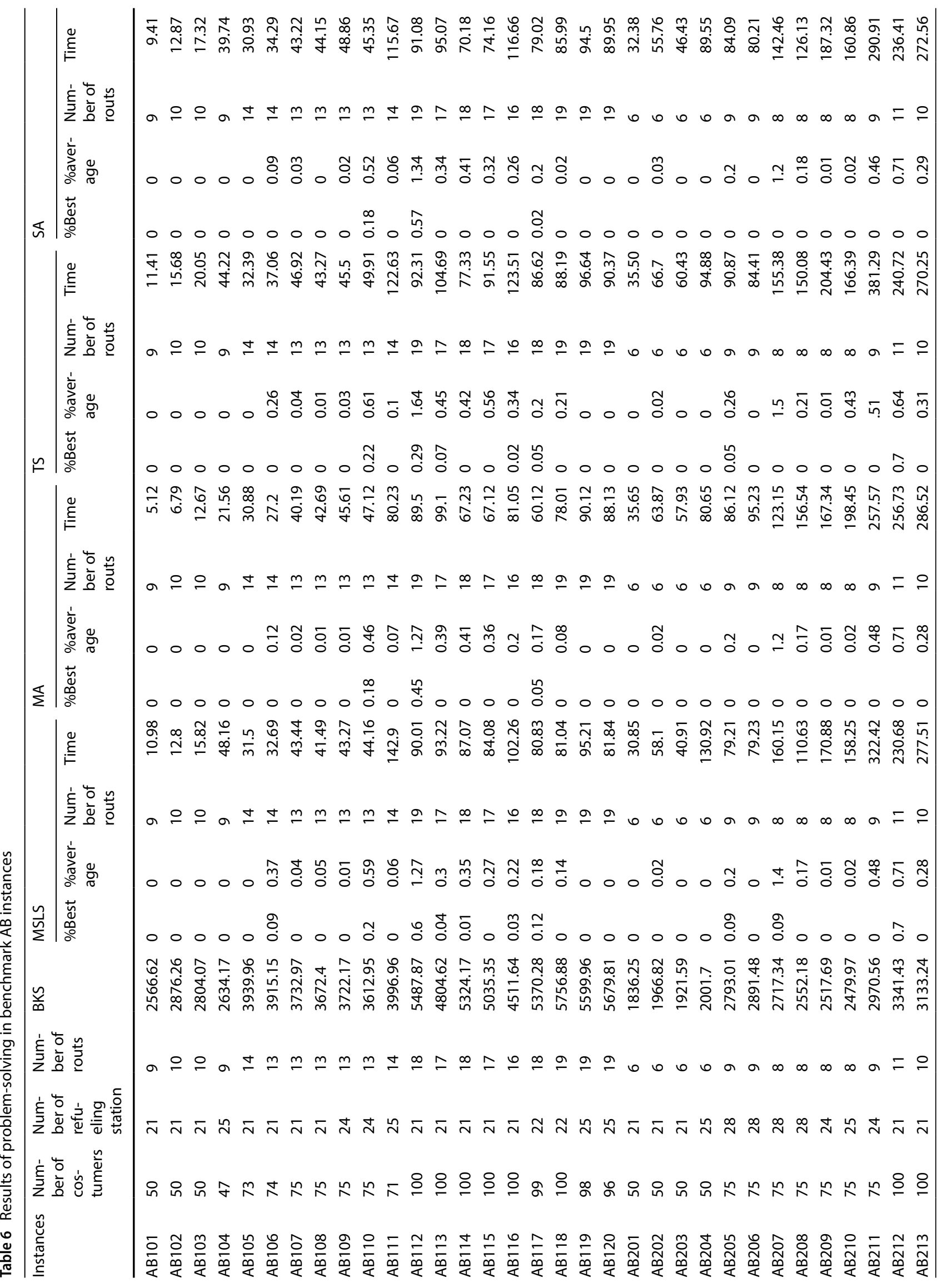




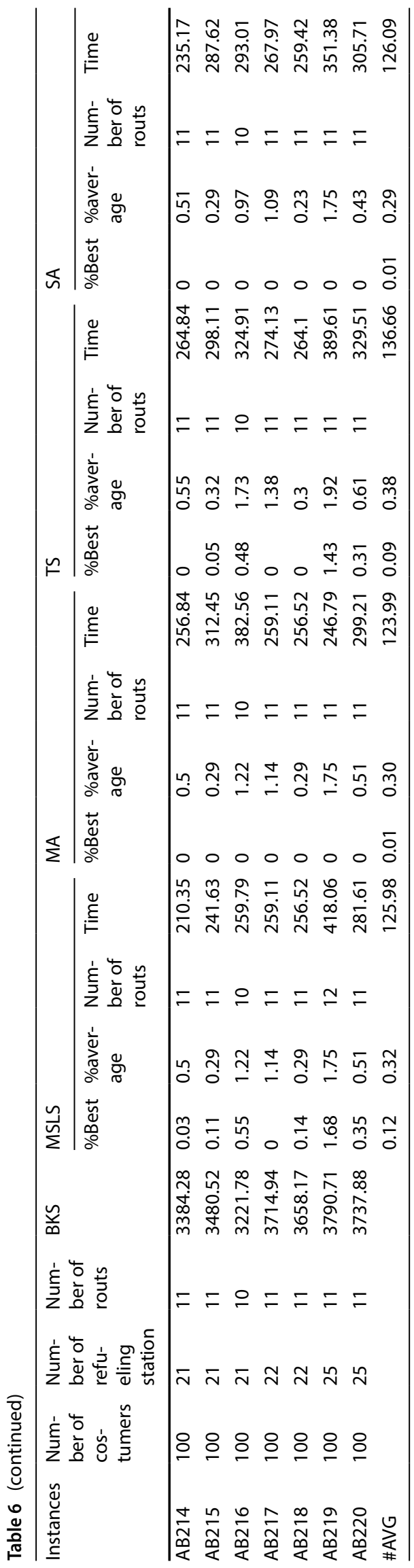

capabilities of the model and compare the discounted and non-discounted mode, 16 instances were generated randomly in small to medium sizes and in addition to the exact method, these instances have been solved with the introduced algorithms. The comparison with the exact method has been completed by taking advantage of GAMS to solve small size problems.

\subsection{Parameter setting}

In meta-heuristic algorithms, it is necessary to adjust the parameters correctly to solve the problem desirably. The tabu search parameters are the number of examined neighbors in each iteration (in), the tabu list length ( $T L$ ), and the number of iterations without any improvement (Lim). The parameters of the SA algorithm are initial temperature $\left(t_{0}\right)$, cooling rate $(\alpha)$, and the maximum number of internal iterations $\left(\right.$ maxit $_{\text {in }}$ ). In this study, the parameters of the proposed algorithms are adjusted by the Taguchi method. This method determines the best possible combination of the input parameters of the algorithm. parameters are tuned using a set of instances with 14 costumers. The results of the TS and SA algorithms parameters adjustment by Taguchi shown in Table 4. In this table $n$ is the number of variables.

\subsection{Numerical examples}

To indicate the effectuality of the proposed algorithms, we employed a set of GVRP instances that were created by Andelmin and Bartolini (2017) [17] by extracting a subset of customers from the larger Erdogan and Miller Hook [2] instances (available at the URL https://www.vrprep.org/ variants/item/g-vrp.html). These instances are divided into two subsets called $A B 1$ and $A B 2$ that each one has 50 to 100 customers and have the same assumptions as the Erdogan and Miller Hook [2] instances. The fuel consumption rate is 0.2 and the capacity of each vehicle's fuel tanks is equal to 60 . The service time of each customer is $30 \mathrm{~min}$, refueling delay is $15 \mathrm{~min}$, and the total permissible time and maximum driving distance of each tour is $11 \mathrm{~h}$ and 300 miles respectively. The difference between $A B 1$ and $A B 2$ is in vehicles speed.In $A B 1$, the vehicles have constant speed of 40 miles per hour, but vehicles speed in $A B 2$ is 60 miles per hour. This difference allows vehicles to have longer routes in $A B 2$ respect to $A B 1$ instances. In these instances, all locations are provided as geographical coordinates with latitude and longitude and the Haversine formula can be used to calculate distances.

Also, to assess the capabilities of the model in discount policies, we generated 16 instances randomly according with the problem's assumption designed by Erdogan and Miller Hook [2] in a network of 300 to 330 miles. The 


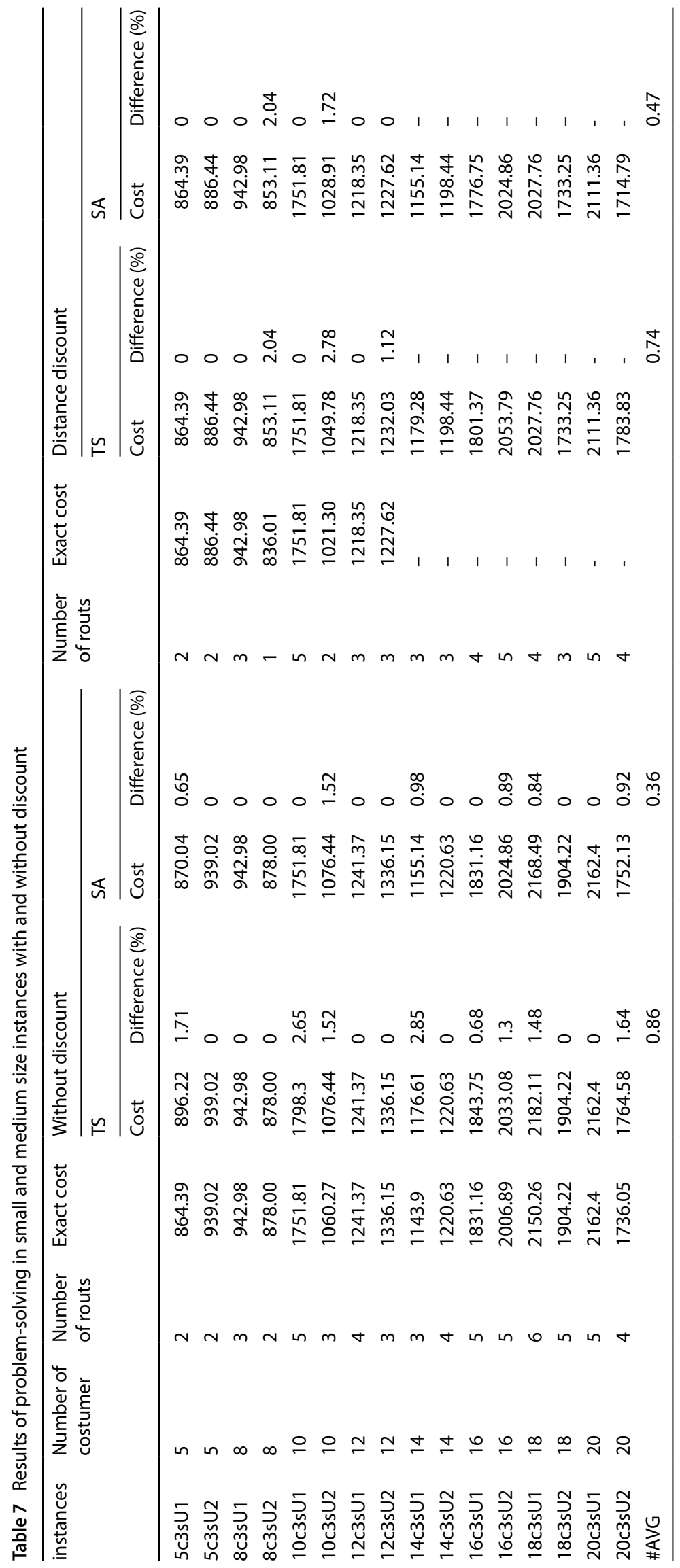




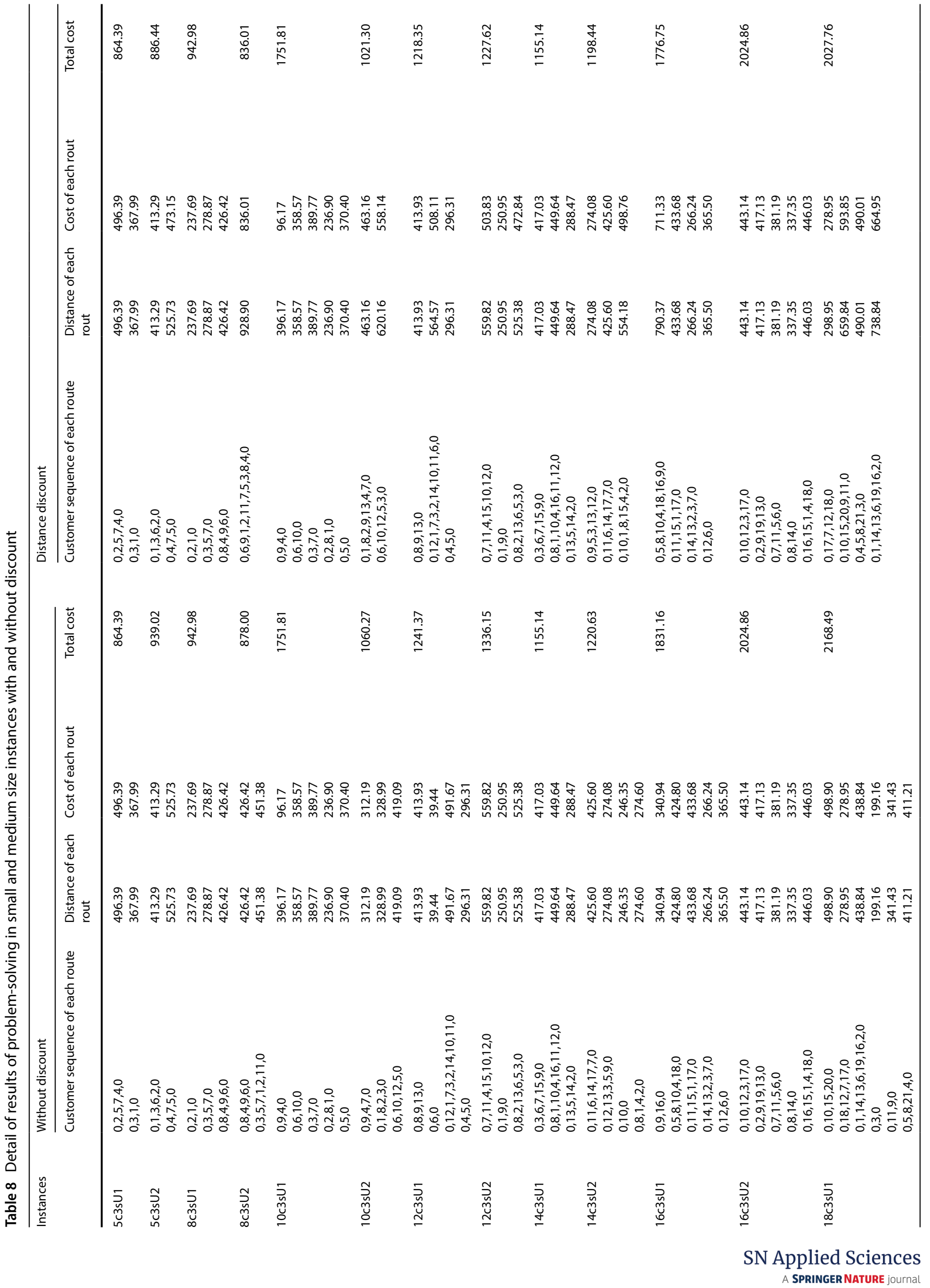




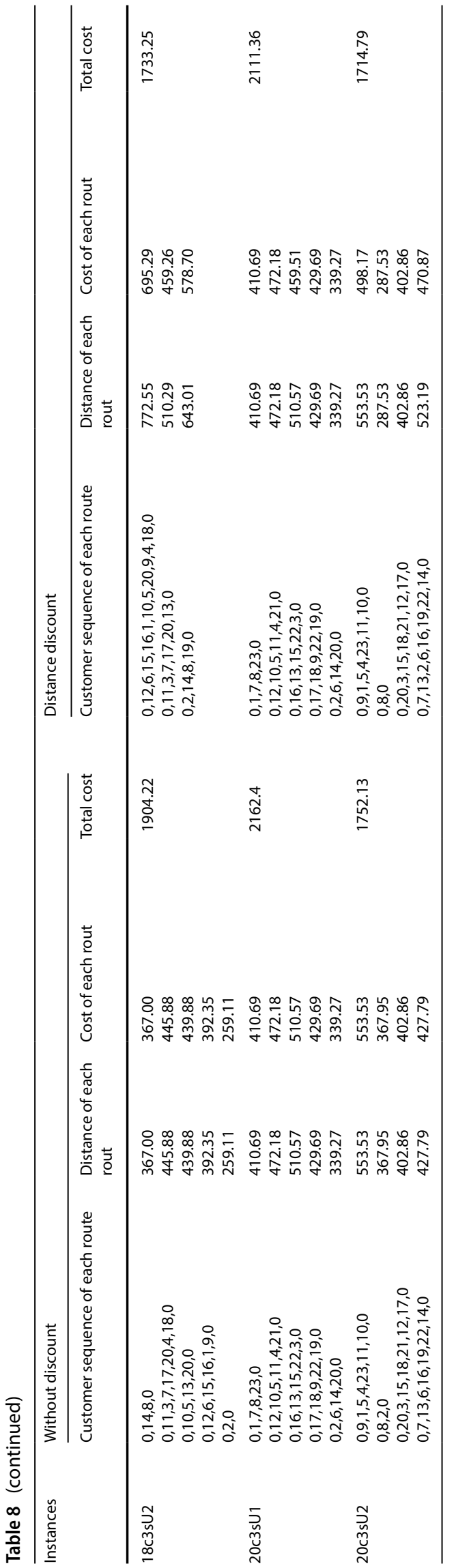

warehouse or distribution center is assumed near the center in all cases and three refueling centers between the distribution center and the grid boundaries in the north, west, and southeast directions.

Customers and refueling stations are distributed uniformly across the network. The fuel consumption rate is 0.15 and the rest of the parameters are similar to the listed problems. Breakpoints, intervals, and discount percentages define in Table 5. The names fo the problems are the same as illustrated examples by Erdogan and Miller Hook [2].In 14C3SU10, the first number shows the number of customers, the second number indicates the number of refueling centers, and the last number represents the example number.

\subsection{Results comparisons on $A B$ instances}

In this section to evaluate the performance and efficiency of the proposed algorithms, each instance has been solved by proposed algorithms for ten times and their results presented in Table 6 in comparison with the best-performing algorithms like MSLS and MA. In this table the first five columns present instance identifiers, number of the customers, number of refueling stations number of routes corresponding to the best solution, and the best-known found results of the literature.

Also, for each algorithm the first two columns show the percentages of average gaps above the BKS values that are computed with respect to the best and average results obtained over 15 runs. The next two columns show the number of routes and the average solving times in seconds. As shown in Table 6, SA outperforms the TS in terms of all the metrics $(0.01$ vs. $0.09,0.29$ vs. $0.38,126.09 \mathrm{~s}$ vs. $136.66 \mathrm{~s})$. Also, the SA algorithm can find 37 BKS out of 40 instances while TS algorithm finds $29 \mathrm{BKS}$. In addition, SA has a lower average gap compared with MA and MSLS and outperforms MSLS in average gap with BKS.

\subsection{Results comparisons on random instances}

To analyze the model and illustrate the effect of the distance discount, 16 samples have been solved by the exact and meta-heuristic methods and their results presented in Table 7. This table illustrates the value of the objective function in different sizes and discount policies. As stated, given that this problem is NP-hard, GAMS would not be able to solve the problems in large-scale problems in an acceptable time. In this comparison, the performance time of the meta-heuristic algorithms has been considered $60 \mathrm{~s}$. Table 8 presented for a detailed view on the effects of discounting. This table demonstrates the sequence of customers, length, and cost of each route and total routing cost in discounted and non-discounted modes. 
Fig. 4 Comparison of SA results in different policies

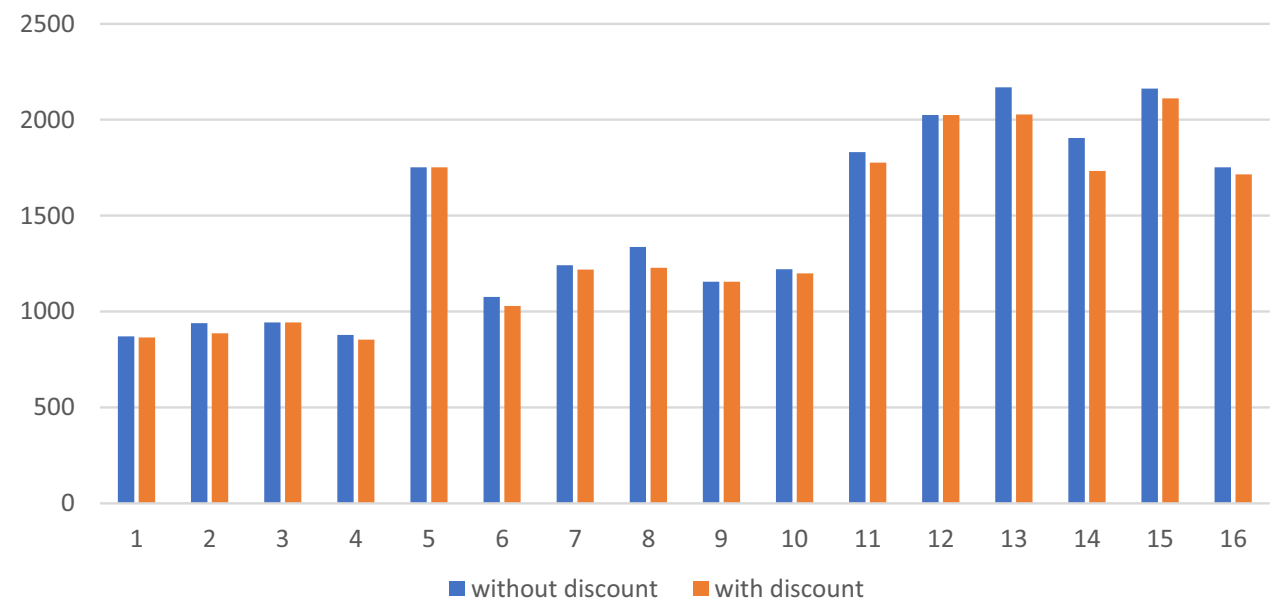

Figure 4 illustrates the difference between total transportation cost in no discount policy and discount policy terms. In this comparison, the results are related to the simulated annealing method, where vertical axis indicates the total transportation cost.

As it turns out, in discounted policy, routes face a reduction in cost, which is normal, but in cases where the limit of maximum time and fuel are not activated, the model seeks longer routes to have a higher discount rate.

\section{Conclusion and future research}

This article investigates a discount strategy in the green vehicle routing problem. In this case, external carriers attempt to gain more customers by offering distance type discounts. We assumed distance intervals in such a way that by increasing distance, the unit distance cost will be decreased. The main goal of the vehicle routing problem is to assign customers to vehicles and reduce the cost of routing and consequently, the entire transportation cost. In this paper, due to the discount offered by the external transportation fleet, we proposed a mix integer linear programming that reduces the total cost of the system.

Forasmuch as this model is recognized as NP-hard, mostly, by increasing customer numbers, the problem complexity will be increased. Accordingly, TS and SA metaheuristic algorithms are used to solve the problem. We solved benchmark instances with these algorithms and compared our results with the results of reviewed algorithms like MSLS and MA that have a significant performance in solving GVRP. Although, both of them have an acceptable performance,comparison shows that SA outperforms the TS in terms of all the metrics. According to the results, in the discount mode, the model seeks to create routes with long paths by assigning greater numbers of customers. The maximum allowed time and fuel limitations perform an essential role in the optimal answer.
So, in the absence of such constraints, the model seeks to form long routes and use the most discount modes, which may not be possible in reality. In our model, when these constraints are allowed, longer routes are selected and are in the discount range, which reduces the overall cost of the organization.

The proposed model and distance discount strategy, in addition to the green routing problem, can be used for a variety of routing issues. Other further research could be: Considering uncertainties and fuzzy parameters; Adding complementary elements to the standard vehicle routing problem such as multiple warehouses, time windows, heterogeneous fleets, etc.; Using other solving methods and metaheuristic algorithms to improve problem-solving especially in large sizes; Implementation of the proposed mathematical model in a real case study.

Acknowledgements We would like to thank the editor-in-chief, subject editor and anonymous reviewers for their constructive and helpful comments on the earlier version of this manuscript.

\section{Compliance with ethical standards}

Conflict of interest The authors declare that there are no conflicts of interest regarding the publication of this paper.

\section{References}

1. Alabas-Uslu C, Dengiz B (2011) A self-adaptive local search algorithm for the classical vehicle routing problem. Expert Syst Appl 38(7):8990-8998. https://doi.org/10.1016/j.eswa.2011.01.116

2. Erdoğan S, Miller-Hooks E (2012) A green vehicle routing problem. Transp Res Part E Logist Transp Rev 48(1):100-114. https:// doi.org/10.1016/j.tre.2011.08.001

3. Li JM, Li AH, Varbanov PS, Liu ZY (2017) Distance potential concept and its applications to the design of regional biomass supply chains and solving vehicle routing problems. J Clean Prod 144:426-436. https://doi.org/10.1016/j.jclepro.2016.12.166

4. Tiwari A, Chang PC (2015) A block recombination approach to solve green vehicle routing problem. Int J Prod Econ 164:379387. https://doi.org/10.1016/j.ijpe.2014.11.003 
5. Sbihi A, Eglese RW (2010) Combinatorial optimization and green logistics. Ann Oper Res 175(1):159-175. https://doi.org/10.1007/ s10479-009-0651-z

6. Lin C, Choy KL, Ho GT, Chung SH, Lam HY (2014) Survey of green vehicle routing problem: past and future trends. Expert Syst Appl 41(4):1118-1138. https://doi.org/10.1016/j. eswa.2013.07.107

7. Schneider M, Stenger A, Goeke D (2014) The electric vehiclerouting problem with time windows and recharging stations. Transp Sci 48(4):500-520. https://doi.org/10.1287/ trsc.2013.0490

8. Trentini A, Mahléné N (2010) Toward a shared urban transport system ensuring passengers \& goods cohabitation. Tema J Land Use Mob Environ. https://doi.org/10.6092/1970-9870/165

9. Ballou RH (2004) Business logistics, supply chain management. Upper Saddle River, Aufl

10. Kara I, Kara BY, Yetis MK (2007) Energy minimizing vehicle routing problem. Int Conf Comb Optim Appl. https://doi. org/10.1007/978-3-540-73556-4_9

11. Xiao Y, Zhao Q, Kaku I, Xu Y (2012) Development of a fuel consumption optimization model for the capacitated vehicle routing problem. Comput Oper Res 39(7):1419-1431. https://doi. org/10.1016/j.cor.2011.08.013

12. Gonçalves F, Cardoso SR, Relvas S, Barbosa-Póvoa APFD (2011) Optimization of a distribution network using electric vehicles: A VRP problem. In: Proceedings of the IO2011-15 Congresso da associação Portuguesa de Investigação Operacional 18-20

13. Conrad RG, Figliozzi MA (2011) The recharging vehicle routing problem. In: Proceedings of the 2011 industrial engineering research conference 1-8

14. Felipe Á, Ortuño MT, Righini G, Tirado G (2014) A heuristic approach for the green vehicle routing problem with multiple technologies and partial recharges. Transp Res Part E Logist Transp Rev 71:111-128. https://doi.org/10.1016/j. tre.2014.09.003

15. Bruglieri M, Mancini S, Pisacane O (2019) The green vehicle routing problem with capacitated alternative fuel stations. Comput Oper Res 112:104759. https://doi.org/10.1016/j.cor.2019.07.017

16. Zhang S, Gajpal Y, Appadoo SS (2018) A meta-heuristic for capacitated green vehicle routing problem. Ann Oper Res 269(1-2):753-771. https://doi.org/10.1007/s10479-017-2567-3

17. Andelmin J, Bartolini E (2017) An exact algorithm for the green vehicle routing problem. Transp Sci 51(4):1288-1303. https:// doi.org/10.1287/trsc.2016.0734

18. Andelmin J, Bartolini E (2019) A multi-start local search heuristic for the green vehicle routing problem based on a multigraph reformulation. Comput Oper Res 109:43-63. https://doi. org/10.1016/j.cor.2019.04.018

19. Peng B, Zhang Y, Gajpal Y, Chen X (2019) A memetic algorithm for the green vehicle routing problem. Sustainability 11(21):6055. https://doi.org/10.3390/su11216055

20. Toptal A (2009) Replenishment decisions under an all-units discount schedule and stepwise freight costs. Eur J Oper Res 198(2):504-510. https://doi.org/10.1016/j.ejor.2008.09.037

21. Tersine RJ, Barman S (1991) Economic inventory/transport lot, sizing with quantity and freight rate discounts. Decis Sci 22(5):1171-1179. https://doi.org/10.1111/j.1540-5915.1991. tb01914.x

22. Sun L, Karwan MH, Gemici-Ozkan B, Pinto JM (2015) Estimating the long-term cost to serve new customers in joint distribution. Comput Ind Eng 80:1-11. https://doi.org/10.1016/j. cie.2014.11.012

23. Gahm C, Brabänder C, Tuma A (2017) Vehicle routing with private fleet, multiple common carriers offering volume discounts, and rental options. Transp Res Part E Logist Transp Rev 97:192216. https://doi.org/10.1016/j.tre.2016.10.010

24. Tsao YC, Lu JC (2012) A supply chain network design considering transportation cost discounts. Transp Res Part E Logist Transp Rev 48(2):401-414. https://doi.org/10.1016/j.tre.2011.10.004

25. Karimi B, Hassanlu MG, Niknamfar AH (2019) An integrated production-distribution planning with a routing problem and transportation cost discount in a supply chain. Assem Autom 39(5):783-802. https://doi.org/10.1108/AA-10-2017-127

26. Stenger A, Schneider M, Goeke D (2013) The prize-collecting vehicle routing problem with single and multiple depots and non-linear cost. EURO J Transp Logist 2:57-87. https://doi. org/10.1007/s13676-013-0022-4

27. Lenstra JK, Kan AR (1981) Complexity of vehicle routing and scheduling problems. Networks 11(2):221-227. https://doi. org/10.1002/net.3230110211

28. Elshaer R, Awad H (2020) A taxonomic review of metaheuristic algorithms for solving the vehicle routing problem and its variants. Comput Ind Eng 140:106242. https://doi.org/10.1016/j. cie.2019.106242

29. Gendreau M, Potvin JY (2010) Handbook of metaheuristics. Springer, New York

30. Glover F, Laguna M (1998) Tabu search. In: Du DZ, Pardalos PM (eds) Handbook of combinatorial optimization. Springer, Boston, MA, pp 2093-2229

31. Qiu M, Fu Z, Eglese R, Tang Q (2018) A Tabu Search algorithm for the vehicle routing problem with discrete split deliveries and pickups. Comput Oper Res 100:102-116. https://doi. org/10.1016/j.cor.2018.07.021

32. Ghosh T, Sengupta S, Chattopadhyay M, Dan P (2011) Metaheuristics in cellular manufacturing: a state-of-the-art review. Int J Ind Eng Comput 2(1):87-122. https://doi.org/10.5267/j.ijiec .2010.04.005

33. Lai DS, Demirag OC, Leung JM (2016) A tabu search heuristic for the heterogeneous vehicle routing problem on a multigraph. Transp Res Part E Logist Transp Rev 86:32-52. https:// doi.org/10.1016/j.tre.2015.12.001

34. Kirkpatrick S, Gelatt CD, Vecchi MP (1983) Optimization by simulated annealing. Science 220(4598):671-680. https://doi. org/10.1126/science.220.4598.671

35. Lee DH, Cao Z, Meng Q (2007) Scheduling of two-transtainer systems for loading outbound containers in port container terminals with simulated annealing algorithm. Int J Prod Econ 107(1):115-124. https://doi.org/10.1016/j.ijpe.2006.08.003

36. Rabbani M, Bosjin S, Yazdanparast R, Saravi N (2018) A stochastic time-dependent green capacitated vehicle routing and scheduling problem with time window, resiliency and reliability: a case study. Decis Sci Lett 7(4):381-394. https://doi.org/10.5267/j. dsl.2018.2.002

37. Mousavi SM, Tavakkoli-Moghaddam R (2013) A hybrid simulated annealing algorithm for location and routing scheduling problems with cross-docking in the supply chain. J Manuf Syst 32(2):335-347. https://doi.org/10.1016/j.jmsy.2012.12.002

38. Birim Ş (2016) Vehicle routing problem with cross docking: a simulated annealing approach. Proc-Soc Behav Sci 235(8):149158. https://doi.org/10.1016/j.sbspro.2016.11.010

39. Borges $\mathrm{P}$, Eid T, Bergseng E (2014) Applying simulated annealing using different methods for the neighborhood search in forest planning problems. Eur J Oper Res 233(3):700-710. https://doi. org/10.1016/j.ejor.2013.08.039

Publisher's Note Springer Nature remains neutral with regard to jurisdictional claims in published maps and institutional affiliations. 